Kragujevac Journal of Mathematics

Volume 44(2) (2020), Pages 181-188.

\title{
NEW UPPER AND LOWER BOUNDS FOR SOME DEGREE-BASED GRAPH INVARIANTS
}

\author{
A. GHALAVAND ${ }^{1}$, A. ASHRAFI ${ }^{1}$, AND I. GUTMAN ${ }^{2}$ \\ Abstract. For a simple graph $G$ with vertex set $V(G)$ and edge set $E(G)$, let \\ $\operatorname{deg}(u)$ be the degree of the vertex $u \in V(G)$. The forgotten index of $G$ and its \\ coindex are defined as $F(G)=\sum_{v \in V(G)} \operatorname{deg}^{3}(v)$ and $\bar{F}(G)=\sum_{u v \notin E(G)}\left[\operatorname{deg}^{2}(u)+\right.$ \\ $\left.\operatorname{deg}^{2}(v)\right]$. New bonds for the first Zagreb index $M_{1}(G)=\sum_{v \in V(G)} \operatorname{deg}(v)^{2}$, forgotten \\ index, and its coindex are obtained.
}

\section{INTRODUCTION}

Throughout this paper, all graphs considered are assumed to be simple, i.e., without directed, weighted, or multiple edges, without self-loops and with a finite number of vertices. Let $G$ be such a graph with vertex set $V(G)=\left\{v_{1}, v_{2}, \ldots, v_{n}\right\}$ and edge set $E(G)$. A graph with $n$ vertices and $m$ edges will be referred to as an $(n, m)$-graph.

By $\operatorname{deg}(v)$ or $\operatorname{deg}_{G}(v)$ is denoted the degree of the vertex $v \in V(G)$. Let $D(G)=$ $\left\{\operatorname{deg}\left(v_{1}\right), \operatorname{deg}\left(v_{2}\right), \ldots, \operatorname{deg}\left(v_{n}\right)\right\}$. If $D(G)=\{r\}$, then $G$ is said to be $r$-regular. If $D(G)=\{r, s\}$, then we say that $G$ is $(r, s)$-biregular. This includes the case of regular graphs if $r=s$. Analogously, if $D(G)=\{r, s, t\}$, then the graph $G$ will be said to be $(r, s, t)$-triregular. Let, in addition, $\Delta=\max _{v \in V(G)} \operatorname{deg}(v)$ and $\delta=\min _{v \in V(G)} \operatorname{deg}(v)$.

The first Zagreb index $M_{1}(G)$ is defined as [13]

$$
M_{1}=M_{1}(G)=\sum_{v \in V(G)} \operatorname{deg}^{2}(v)=\sum_{u v \in E(G)}[\operatorname{deg}(u)+\operatorname{deg}(v)] .
$$

It is the oldest and most studied degree-based graph invariant; details of its mathematical theory and chemical applications can be found in the surveys $[5,11,17]$.

Key words and phrases. Degree (of vertex), coindex, forgotten index, F-index, Zagreb index. 2010 Mathematics Subject Classification. Primary: 05C07. Secondary: 05C90.

DOI 10.46793/KgJMat2002.181G

Received: March 6, 2018.

Accepted: March 22, 2018. 
In the paper [13], $M_{1}$ was used for designing approximate expressions for total $\pi$-electron energy. In the same paper, also the sum of cubes of vertex degrees $(F)$ was used for the same purpose. However, whereas $M_{1}$ eventually gained much popularity $[5,11,17]$, no attention was paid to $F$. Only more than forty years later, the invariant $F$ attracted some interest, thanks to the discovery of its applicability in physical chemistry [4]. For this reason it was named forgotten index and is defined as [4]:

$$
F=F(G)=\sum_{v \in V(G)} \operatorname{deg}(v)^{3}=\sum_{u v \in E(G)}\left[\operatorname{deg}(u)^{2}+\operatorname{deg}(v)^{2}\right]
$$

In the last few years, numerous mathematical studies of the forgotten index have been published, see $[1-3,6,7,10,12,16]$.

Some of pharmacological applications of the $F$-index were also attempted [15].

Both $M_{1}$ and $F$ are special cases of the so-called first general Zagreb index, defined as

$$
M_{1}^{\alpha}=M_{1}^{\alpha}(G)=\sum_{u \in V(G)} \operatorname{deg}(u)^{\alpha}=\sum_{u v \in E(G)}\left[\operatorname{deg}(u)^{\alpha-1}+\operatorname{deg}(v)^{\alpha-1}\right]
$$

where $\alpha$ is an arbitrary real number $[15,18]$.

The coindex of $M_{1}^{\alpha}$ is defined as [18]

$$
\overline{M_{1}^{\alpha}}(G)=\sum_{\substack{u v \notin E(G) \\ u \neq v}}\left[\operatorname{deg}(u)^{\alpha-1}+\operatorname{deg}(v)^{\alpha-1}\right] .
$$

The special case of this expressions for $\alpha=3$ is the coindex of the forgotten index $[8,14]$

$$
\bar{F}(G)=\sum_{\substack{u v \notin E(G) \\ u \neq v}}\left[\operatorname{deg}(u)^{2}+\operatorname{deg}(v)^{2}\right]
$$

\section{Main Results}

We first state results that improve those reported in [12]. Denote by $\bar{G}$ the complement of the graph $G$.

Theorem 2.1. Let $G$ be an $(n, m)$-graph. Then

$$
F(G)+F(\bar{G})=n^{4}+M_{1}(G)(3 n-3)-2 m\left(3 n^{2}-6 n+3\right)-n\left(3 n^{2}-3 n+1\right)
$$

and

$$
\begin{aligned}
F(G) \times F(\bar{G})= & n^{4} F(G)+(3 n-3) F(G) M_{1}(G)-2 m\left(3 n^{2}-6 n+3\right) F(G) \\
& -n\left(3 n^{2}-3 n+1\right) F(G)-F(G)^{2} .
\end{aligned}
$$


Proof. By definition of a graph complement, we have

$$
\begin{aligned}
F(\bar{G})= & \sum_{u \in V(G)} \operatorname{deg}_{\bar{G}}(u)^{3}=\sum_{u \in V(G)}\left[n-1-\operatorname{deg}_{G}(u)\right]^{3} \\
= & \sum_{u \in V(G)}\left[n^{3}+\operatorname{deg}_{G}(u)^{2}(3 n-3)-\operatorname{deg}_{G}(u)\left(3 n^{2}-6 n+3\right)\right. \\
& \left.-3 n^{2}+3 n-1-\operatorname{deg}_{G}(u)^{3}\right] \\
= & n^{4}+M_{1}(G)(3 n-3)-2 m\left(3 n^{2}-6 n+3\right)-n\left(3 n^{2}-3 n+1\right)-F(G) .
\end{aligned}
$$

Theorem 2.2. Let $G$ be an $(n, m)$-graph. Then

$$
F(G) \leq n \Delta^{3}+3 \Delta M_{1}(G)-6 m \Delta^{2} \text { and } F(G) \geq n \delta^{3}+3 \delta M_{1}(G)-6 m \delta^{2},
$$

with equalities if and only if $G$ is regular.

Proof. Define an auxiliary function $Y_{1}(G)=\sum_{u \in V(G)}[\operatorname{deg}(u)-k]^{3}$, where $k$ is a real number. Then,

$$
\begin{aligned}
Y_{1}(G) & =\sum_{u \in V(G)}\left[\operatorname{deg}(u)^{3}-k^{3}-3 \operatorname{deg}(u)^{2} k+3 \operatorname{deg}(u) k^{2}\right] \\
& =F(G)-n k^{3}-3 k M_{1}(G)+6 m k^{2} .
\end{aligned}
$$

If $k=\Delta$, then $Y_{1}(G) \leq 0$ and $F(G) \leq n \Delta^{3}+3 \Delta M_{1}(G)-6 m \Delta^{2}$. For $k=\delta, Y_{1}(G) \geq 0$ and $F(G) \geq n \delta^{3}+3 \delta M_{1}(G)-6 m \delta^{2}$. The equalities hold if and only if $G$ is regular.

Theorem 2.3. Let $G$ be an $(n, m)$-graph. Then

$$
F(G) \geq M_{1}(G)(\delta+2 \Delta)-\Delta^{2}(2 m-n \delta)-4 m \Delta \delta
$$

and

$$
F(G) \leq M_{1}(G)(\Delta+2 \delta)-\delta^{2}(2 m-n \Delta)-4 m \delta \Delta
$$

with equalities if and only if $G$ is $(\Delta, \delta)$-biregular.

Proof. Define $Y_{2}(G)=\sum_{u \in V(G)}[\operatorname{deg}(u)-k]^{2}[\operatorname{deg}(u)-h]$, where $k$ and $h$ are real numbers. Then,

$$
\begin{aligned}
Y_{2}(G) & =\sum_{u \in V(G)}\left[\operatorname{deg}(u)^{2}+k^{2}-2 \operatorname{deg}(u) k\right][\operatorname{deg}(u)-h] \\
& =\sum_{u \in V(G)}\left[\operatorname{deg}(u)^{3}-\operatorname{deg}(u)^{2} h+\operatorname{deg}(u) k^{2}-k^{2} h-2 \operatorname{deg}(u)^{2} k+2 \operatorname{deg}(u) k h\right] \\
& =F(G)-M_{1}(G)(h+2 k)+k^{2}(2 m-n h)+4 m k h .
\end{aligned}
$$

If $k=\Delta$ and $h=\delta$, then $Y_{2}(G) \geq 0$ and $F(G) \geq M_{1}(G)(\delta+2 \Delta)-\Delta^{2}(2 m-n \delta)-$ $4 m \Delta \delta$. For $k=\delta$ and $h=\Delta$, we have $Y_{2}(G) \leq 0$ and $F(G) \leq M_{1}(G)(\Delta+2 \delta)-$ $\delta^{2}(2 m-n \Delta)-4 m \delta \Delta$. The equalities hold if and only if $G$ is $(\Delta, \delta)$-biregular.

Theorem 2.4. Let $G$ be an $(n, m)$-graph. Then $F(G) \geq 2\left[M_{1}(G)+m-n\right]$. If $G$ is connected, then equality holds if and only if $G \cong P_{n}$ or $G \cong C_{n}$. 
Proof. Define the auxiliary function $Y_{3}(G)=\sum_{u \in V(G)}\left[\operatorname{deg}(u)^{2}-1\right][\operatorname{deg}(u)-2]$ and note that $Y_{3}(G)=0$ if and only if $\Delta(G) \leq 2$. In case of connected graphs, this will occur if either $G \cong P_{n}$ or $G \cong C_{n}$.

Now,

$$
\begin{aligned}
Y_{3}(G) & =\sum_{u \in V(G)}\left[\operatorname{deg}(u)^{3}-2 \operatorname{deg}(u)^{2}-\operatorname{deg}(u)+2\right] \\
& =F(G)-2 M_{1}(G)-2 m+2 n .
\end{aligned}
$$

Since $Y_{3}(G) \geq 0, F(G) \geq 2\left[M_{1}(G)+m-n\right]$ with equality for connected graphs if and only if $G \cong P_{n}$ or $G \cong C_{n}$.

Theorem 2.5. Let $G$ be an $(n, m)$-graphs. Then

$$
F(G) \leq(3 \Delta-3) M_{1}(G)-2 m\left(3 \Delta^{2}-6 \Delta+2\right)+n \Delta(\Delta-1)(\Delta-2)
$$

and

$$
F(G) \geq(3 \delta+3) M_{1}(G)-2 m\left(3 \delta^{2}+6 \delta+2\right)+n \delta(\delta+1)(\delta+2) .
$$

The equalities holds if and only if $G$ is $(\delta, \delta+1, \delta+2)$-triregular.

Proof. Define $Y_{4}(G)=\sum_{u \in V(G)}[\operatorname{deg}(u)-a][\operatorname{deg}(u)-b][\operatorname{deg}(u)-c]$, where $a, b$, and $c$ are real numbers. Then,

$$
\begin{aligned}
Y_{4}(G) & =\sum_{u \in V(G)}\left[\operatorname{deg}(u)^{3}-\operatorname{deg}(u)^{2}(a+b+c)+\operatorname{deg}(u)(a b+a c+b c)-a b c\right] \\
& =F(G)-(a+b+c) M_{1}(G)+2 m(a b+a c+b c)-n a b c .
\end{aligned}
$$

If $a=\Delta, b=\Delta-1$ and $c=\Delta-2$, then $Y_{4}(G) \leq 0$ and $F(G) \leq(3 \Delta-3) M_{1}(G)-$ $2 m\left(3 \Delta^{2}-6 \Delta+2\right)+n \Delta(\Delta-1)(\Delta-2)$. For $a=\delta, b=\delta+1$ and $c=\delta+2, Y_{4}(G) \geq 0$ and $F(G) \geq(3 \delta+3) M_{1}(G)-2 m\left(3 \delta^{2}+6 \delta+2\right)+n \delta(\delta+1)(\delta+2)$. The equalities hold if and only if $G$ is $(\delta, \delta+1, \delta+2)$-triregular.

For the sake of completeness, we mention here a result from [18].

Theorem 2.6. [18] Let $G$ be an $(n, m)$-graph. Then for $\alpha \geq 1$,

$$
\overline{M_{1}^{\alpha+1}}(G)=(n-1) M_{1}^{\alpha}(G)-M_{1}^{\alpha+1}(G) .
$$

Theorem 2.7. Let $G$ be an $(n, m)$-graph. Then

$$
\bar{F}(G) \geq 2 m\left[2 \Delta(n-1)+3 \Delta^{2}\right]-n\left[(n-1) \Delta^{2}+\Delta^{3}\right]-3 \Delta M_{1}(G) .
$$

The equality holds if and only if $G$ is regular.

Proof. Define

$$
Y_{5}(G)=(n-1) \sum_{u \in V(G)}[\operatorname{deg}(u)-\Delta]^{2}-\sum_{u \in V(G)}[\operatorname{deg}(u)-\Delta]^{3} .
$$


Then,

$$
\begin{aligned}
Y_{5}(G)= & (n-1) \sum_{u \in V(G)}\left[\operatorname{deg}(u)^{2}+\Delta^{2}-2 \Delta \operatorname{deg}(u)\right] \\
& -\sum_{u \in V(G)}\left[\operatorname{deg}(u)^{3}-\Delta^{3}-3 \Delta \operatorname{deg}(u)^{2}+3 \Delta^{2} \operatorname{deg}(u)\right] \\
= & (n-1) M_{1}(G)-F(G)+n\left[(n-1) \Delta^{2}+\Delta^{3}\right] \\
& -2 m\left[2 \Delta(n-1)+3 \Delta^{2}\right]+3 \Delta M_{1}(G) .
\end{aligned}
$$

Since $Y_{5}(G) \geq 0$, one can see that

$$
(n-1) M_{1}(G)-F(G) \geq 2 m\left[2 \Delta(n-1)+3 \Delta^{2}\right]-n\left[(n-1) \Delta^{2}+\Delta^{3}\right]-3 \Delta M_{1}(G) .
$$

The equality holds if and only if $G$ is a regular graph. Therefore, by Theorem 2.6,

$$
\bar{F}(G) \geq 2 m\left[2 \Delta(n-1)+3 \Delta^{2}\right]-n\left[(n-1) \Delta^{2}+\Delta^{3}\right]-3 \Delta M_{1}(G)
$$

with equality if and only if $G$ is regular.

Theorem 2.8. Let $G$ be an $(n, m)$-graph. Then

$$
\begin{aligned}
\bar{F}(G) \geq & 2 m\left[(n-1)(2 \Delta-1)+\Delta^{2}+2 \Delta(\Delta-1)\right]-M_{1}(G)(3 \Delta-1) \\
& -n\left[(n-1) \Delta(\Delta-1)+\Delta^{2}(\Delta-1)\right] .
\end{aligned}
$$

The equality holds if and only if $G$ is $(\Delta, \Delta-1)$-biregular.

Proof. We define the auxiliary function

$$
\begin{aligned}
Y_{6}(G)= & (n-1) \sum_{u \in V(G)}[\operatorname{deg}(u)-\Delta][\operatorname{deg}(u)-(\Delta-1)] \\
& -\sum_{u \in V(G)}[\operatorname{deg}(u)-\Delta]^{2}[\operatorname{deg}(u)-(\Delta-1)] .
\end{aligned}
$$

Then,

$$
\begin{aligned}
Y_{6}(G)= & (n-1) \sum_{u \in V(G)}\left[\operatorname{deg}(u)^{2}-\operatorname{deg}(u)(2 \Delta-1)+\Delta(\Delta-1)\right] \\
& -\sum_{u \in V(G)}\left[\operatorname{deg}(u)^{3}-\operatorname{deg}(u)^{2}(3 \Delta-1)+\operatorname{deg}(u) \Delta^{2}\right. \\
& \left.-\Delta^{2}(\Delta-1)+2 \operatorname{deg}(u) \Delta(\Delta-1)\right] \\
= & (n-1) M_{1}(G)-2 m(n-1)(2 \Delta-1)+n(n-1) \Delta(\Delta-1) \\
& -F(G)+M_{1}(G)(3 \Delta-1)-2 m \Delta^{2}+n \Delta^{2}(\Delta-1)-4 m \Delta(\Delta-1) \\
= & (n-1) M_{1}(G)-F(G)-2 m\left[(n-1)(2 \Delta-1)+\Delta^{2}+2 \Delta(\Delta-1)\right] \\
& +n\left[(n-1) \Delta(\Delta-1)+\Delta^{2}(\Delta-1)\right]+M_{1}(G)(3 \Delta-1) .
\end{aligned}
$$


Since $Y_{6}(G) \geq 0$,

$$
\begin{aligned}
(n-1) M_{1}(G)-F(G) \geq & 2 m\left[(n-1)(2 \Delta-1)+\Delta^{2}+2 \Delta(\Delta-1)\right] \\
& -n\left[(n-1) \Delta(\Delta-1)+\Delta^{2}(\Delta-1)\right]-(3 \Delta-1) M_{1}(G),
\end{aligned}
$$

with equality if and only if $G$ is a $(\Delta, \Delta-1)$-biregular graph. We now apply Theorem 2.6 to show that

$$
\begin{aligned}
\bar{F}(G) \geq & 2 m\left[(n-1)(2 \Delta-1)+\Delta^{2}+2 \Delta(\Delta-1)\right] \\
& -n\left[(n-1) \Delta(\Delta-1)+\Delta^{2}(\Delta-1)\right]-(3 \Delta-1) M_{1}(G)
\end{aligned}
$$

with equality if and only if $G$ is $(\Delta, \Delta-1)$-biregular.

Theorem 2.9. Let $G$ be an $(n, m)$-graph. Then

$$
\bar{F}(G) \leq 2 m\left[(n-1)(\delta+\Delta)+\Delta^{2}+2 \Delta \delta\right]-n\left[(n-1) \Delta \delta+\Delta^{2} \delta\right]-(\delta+2 \Delta) M_{1}(G) .
$$

The equality holds if and only if $G$ is $(\Delta, \delta)$-biregular.

Proof. Define the function

$$
Y_{7}(G)=(n-1) \sum_{u \in V(G)}[\operatorname{deg}(u)-\Delta][\operatorname{deg}(u)-\delta]-\sum_{u \in V(G)}[\operatorname{deg}(u)-\Delta]^{2}[\operatorname{deg}(u)-\delta] .
$$

Then,

$$
\begin{aligned}
Y_{7}(G)= & (n-1) \sum_{u \in V(G)}\left[\operatorname{deg}(u)^{2}-\operatorname{deg}(u)(\delta+\Delta)+\Delta \delta\right] \\
& -\sum_{u \in V(G)}\left[\operatorname{deg}(u)^{3}-\operatorname{deg}(u)^{2}(\delta+2 \Delta)+\operatorname{deg}(u) \Delta^{2}-\Delta^{2} \delta+2 \operatorname{deg}(u) \Delta \delta\right] \\
= & (n-1) M_{1}(G)-2 m(n-1)(\delta+\Delta)+n(n-1) \Delta \delta \\
- & F(G)+M_{1}(G)(\delta+2 \Delta)-2 m \Delta^{2}+n \Delta^{2} \delta-4 m \Delta \delta \\
= & (n-1) M_{1}(G)-F(G)-2 m\left[(n-1)(\delta+\Delta)+\Delta^{2}+2 \Delta \delta\right] \\
& +n\left[(n-1) \Delta \delta+\Delta^{2} \delta\right]+(\delta+2 \Delta) M_{1}(G) .
\end{aligned}
$$

Since $Y_{7}(G) \leq 0$,

$$
\begin{aligned}
(n-1) M_{1}(G)-F(G) \leq & 2 m\left[(n-1)(\delta+\Delta)+\Delta^{2}+2 \Delta \delta\right] \\
& -n\left[(n-1) \Delta \delta+\Delta^{2} \delta\right]-(\delta+2 \Delta) M_{1}(G),
\end{aligned}
$$

and the equality holds if and only if $G$ is a $(\Delta, \delta)$-biregular graph. We now apply Theorem 2.6 to show that,

$$
\bar{F}(G) \leq 2 m\left[(n-1)(\delta+\Delta)+\Delta^{2}+2 \Delta \delta\right]-n\left[(n-1) \Delta \delta+\Delta^{2} \delta\right]-(\delta+2 \Delta) M_{1}(G),
$$

with equality holding if and only if $G$ is $(\Delta, \delta)$-biregular.

Theorem 2.10. Let $G$ be an $(n, m)$-graph. Then the following holds. 
(a) $M_{1}(G) \leq 2 m(\delta+\Delta)-n \Delta \delta$, with equality if and only if $G$ is $(\Delta, \delta)$-biregular.

(b) $M_{1}(G) \geq 2 m(2 \Delta-1)-n \Delta(\Delta-1)$ and $M_{1}(G) \geq 2 m(2 \delta+1)-n \delta(\delta+1)$. The equalities holds if and only if $G$ is $(\delta, \delta+1)$-biregular.

(c) Let $r$ be a real number. Then $M_{1}(G) \geq 4 m a-n r^{2}$, with equality if and only if $G$ is an r-regular graph.

Proof. Consider the function $Y_{8}(G)=\sum_{u \in V(G)}[\operatorname{deg}(u)-a][\operatorname{deg}(u)-b]$, where $a$ and $b$ are real numbers. Then we have,

$$
\begin{aligned}
Y_{8}(G) & =\sum_{u \in V(G)}\left[\operatorname{deg}(u)^{2}-\operatorname{deg}(u) b-\operatorname{deg}(u) a+a b\right] \\
& =M_{1}(G)-2 m(a+b)+n a b .
\end{aligned}
$$

If $a=\Delta$ and $b=\delta$, then $Y_{8}(G) \leq 0$ and $M_{1}(G) \leq 2 m(\delta+\Delta)-n \Delta \delta$. Now the equality holds if and only if $G$ is a $(\Delta, \delta)$-biregular graph. This completes the part (a).

Suppose that $a=\Delta$ and $b=\Delta-1$. Then $Y_{8}(G) \geq 0$ and $M_{1}(G) \geq 2 m(2 \Delta-1)-$ $n \Delta(\Delta-1)$. For $a=\delta$ and $b=\delta+1, Y_{8}(G) \geq 0$ and $M_{1}(G) \geq 2 m(2 \delta+1)-n \delta(\delta+1)$. The equalities hold if and only if $G$ is $(\delta, \delta+1)$-biregular, which completes the proof of part (b).

Finally, assume that $a=b=r$. Then $Y_{8}(G) \geq 0$ and $M_{1}(G) \geq 4 m a-n r^{2}$. The equality holds if and only if $G$ is $r$-regular.

Acknowledgements. The research of the first two authors was partially supported by the University of Kashan under grant no 364988/111.

\section{REFERENCES}

[1] H. Abdo, D. Dimitrov and I. Gutman, On extremal trees with respect to the F-index, Kuwait J. Sci. 44(4) (2017), 1-8.

[2] S. Akhter and M. Imran, Computing the forgotten topological index of four operations on graphs, AKCE Int. J. Graphs Comb. 14 (2017), 70-79.

[3] S. Akhter, M. Imran and M. R. Farahani, Extremal unicyclic and bicyclic graphs with respect to the F-index, AKCE Int. J. Graphs Comb. 14 (2017), 80-91.

[4] B. Furtula and I. Gutman, A forgotten topological index, J. Math. Chem. 53 (2015), 1184-1190.

[5] B. Borovićanin, K. C. Das, B. Furtula and I. Gutman, Bounds for Zagreb indices, MATCH Commun. Math. Comput. Chem. 78 (2017), 17-100.

[6] Z. Che and Z. Chen, Lower and upper bounds of the forgotten topological index, MATCH Commun. Math. Comput. Chem. 76 (2016), 635-648.

[7] N. De, S. M. Abu Nayeem and A. Pal, F-index of some graph operations, Discrete Math. Algorithms Appl. 8 (2016), ID 1650025.

[8] N. De, S. M. Abu Nayeem and A. Pal, The F coindex of some graph operations, Springer Plus 5 (2016), Paper ID 221.

[9] W. Gao, M. K. Siddiqui, M. Imran, M. K. Jamil and M. R. Farahani, Forgotten topological index of chemical structure in drugs, Saudi Pharma. J. 24 (2016), 258-264.

[10] S. Ghobadi and M. Ghorbaninejad, The forgotten topological index of four operations on some special graphs, Bulletin of Mathematical Sciences and Applications 16 (2016), 89-95. 
[11] I. Gutman and K. C. Das, The first Zagreb index 30 years after, MATCH Commun. Math. Comput. Chem. 50 (2004), 83-92.

[12] I. Gutman, A. Ghalavand, T. Dehghan-Zadeh and A. R. Ashrafi, Graphs with smallest forgotten index, Iranian Journal of Mathematical Chemistry 8 (2017), 259-273.

[13] I. Gutman and N. Trinajstić, Graph theory and molecular orbitals. Total $\pi$-electron energy of alternant hydrocarbons, Chemical Physics Letters 17 (1972), 535-538.

[14] A. Khaksari and M. Ghorbani, On the forgotten topological index, Iranian Journal of Mathematical Chemistry 8 (2017), 327-338.

[15] X. Li and H. Zhao, Trees with the first three smallest and largest generalized topological indices, MATCH Commun. Math. Comput. Chem. 50 (2004), 57-62.

[16] I. Ž. Milovanović, E. I. Milovanović, I. Gutman and B. Furtula, Some inequalities for the forgotten topological index, International Journal of Applied Graph Theory 1 (2017), 1-15.

[17] S. Nikolić, G. Kovačević, A. Miličević and N. Trinajstić, The Zagreb indices 30 years after, Croatica Chemica Acta 76 (2003), 113-124.

[18] S. Zhang and H. Zhang, Unicyclic graphs with the first three smallest and largest first general Zagreb index, MATCH Commun. Math. Comput. Chem. 55 (2006), 427-438.

\footnotetext{
${ }^{1}$ Department of Pure Mathematics, Faculty of Mathematical Science, UNIVERSITY OF KASHAN, KASHAN 87317-51167, I. R. IRAN

Email address: ali.ghalavand.kh@gmail.com

Email address: ashrafi@kashanu.ac.ir

${ }^{2}$ Faculty of Science, UNIVERSITY OF KRAGUJEVAC, 34000 Kragujevac, Serbia

Email address: gutman@kg.ac.rs
} 